\title{
Die Rolle des Selbstkonzepts im Mathematikstudium - Wie fit fühlen sich Studierende in Mathematik?
}

\author{
Stefanie Rach $(\mathbb{D}) \cdot$ Stefan Ufer $\cdot$ Timo Kosiol
}

Eingegangen: 23. Mai 2020 / Überarbeitet: 30. April 2021 / Angenommen: 13. Juli 2021 / Online publiziert: 4. November 2021

(C) Der/die Autor(en) 2021

Zusammenfassung Das mathematische Selbstkonzept als Einschätzung der eigenen Fähigkeiten ist relevant für erfolgreiche Lernprozesse. Am Übergang in ein Mathematikstudium ändert sich die Domäne, sodass eine Schul- von einer universitären Mathematik unterschieden werden kann. In diesem Beitrag schlagen wir ein dreidimensionales Modell vor, das ein allgemeines, ein schulisches und ein universitäres mathematisches Selbstkonzept ausdifferenziert. Anhand einer Studie mit 202 Mathematikstudierenden (Fach bzw. Lehramt) geben wir Hinweise für die Güte der neu entwickelten Fragebogenskalen. Schon zu Studienbeginn kann ein Selbstkonzept in Bezug auf die Schulmathematik von einem Selbstkonzept in Bezug auf die universitäre Mathematik unterschieden werden. Die Entwicklung des mathematischen Selbstkonzepts in den ersten Wochen kann durch die differenzierten Facetten präziser beschrieben werden: Während die schulische Facette im Mittel stabil bleibt, verringert sich das universitäre Selbstkonzept, insbesondere bei den Lehramtsstudierenden. Das universitäre und das allgemeine mathematische Selbstkonzept prädizieren die Studienzufriedenheit positiv. Implikationen aus der differenzierten Messung werden diskutiert.

Schlüsselwörter Mathematisches Selbstkonzept $\cdot$ Studieneingangsphase $\cdot$ Struktur des Selbstkonzepts · Entwicklung des Selbstkonzepts

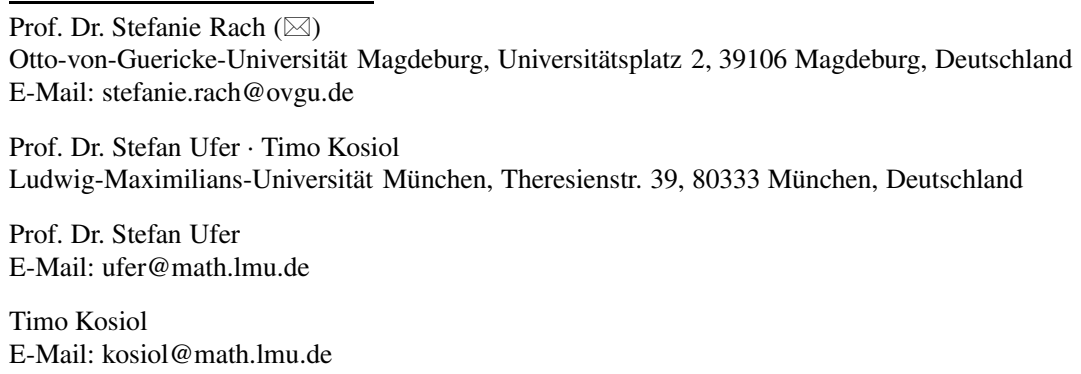




\title{
The role of self-concept when studying mathematics-Do students feel fit in mathematics?
}

\begin{abstract}
The mathematical self-concept as the individual perception of skills is an important factor during learning. At the transition into a mathematics study program, the domain changes so that a school mathematics can be distinguished from a university mathematics. In this contribution, we present a three-dimensional model of mathematical self-concept, which differentiate between a general, a schoolbased, and a university-based mathematical self-concept. Based on a study with 202 students (in a bachelor or teacher education program), we indicate the quality of this new questionnaire for measuring three different self-concept facets. By these differentiated scales, we can deeply describe the development of mathematical selfconcept during the first semester weeks: Whereas the mean-level of school-based mathematical self-concept stays stable, the university mathematical self-concept, especially of teacher education students, decreases. The general and the universitybased mathematical self-concept relates positively to study satisfaction in the middle of the first semester. We discuss implications of the differentiated measures of selfconcept.
\end{abstract}

Keywords Development of self-concept - Mathematical self-concept - Structure of self-concept $\cdot$ Study entrance phase

\section{Einleitung}

Der Übergang in ein Mathematikstudium ist für Studierende mit großen Herausforderungen verbunden. Diese führen bei $54 \%$ der Studierenden zu einem Studienabbruch (Neugebauer et al. 2019), häufig bereits in der Studieneingangsphase (Dieter 2012). Als eine mögliche Ursache für einen Studienabbruch wird unter anderem ein ungünstiges Selbstkonzept angenommen (di Martino und Gregorio 2019).

Das Konstrukt Selbstkonzept beschreibt die retrospektive Einschätzung der eigenen Fähigkeiten (Marsh et al. 2019). Es wird als hierarchisch organisiert angenommen, das sich in verschiedene Facetten gliedert (Shavelson et al. 1976). Diese Facetten repräsentieren die Einschätzung der eigenen Fähigkeiten entsprechend einer spezifischen Domäne, z. B. Mathematik oder Englisch (Jansen et al. 2020). Empirische Studien, vor allem im schulischen Kontext, zeigen, dass das mathematische Selbstkonzept den Lernerfolg positiv beeinflusst (z. B. Feng et al. 2018; Köller et al. 2019). Die Wirkung des Selbstkonzepts auf den Lernerfolg lässt sich nach Erwartungs-Wert-Modellen mit einer höheren Erfolgserwartung erklären, die wiederum die Leistung in Lernprozessen positiv beeinflusst (Eccles und Wigfield 2002, 2020). Der Effekt des mathematischen Selbstkonzepts auf die mathematische Leistung wird partiell auch durch das Engagement mediiert, das bei der Bearbeitung von Mathematikaufgaben investiert wird (Cai et al. 2018).

Da Selbstkonzepte die Einschätzungen der eigenen Fähigkeiten hinsichtlich einer Domäne beschreiben, spielt es eine Rolle, mit welcher Domäne ein Lernender eine spezifische Lernsituation verknüpft. Im Kontext des Übergangs in ein Mathe- 
matikstudium in der hier präsentierten Studie ist diese Domäne nicht eindeutig, denn beim Übergang von der Schule zur Hochschule wandelt sich die Domäne Mathematik: Während in der Schulmathematik ein Schwerpunkt auf der Anwendung mathematischer Inhalte auf außermathematische Probleme liegt, steht in der Hochschule die wissenschaftliche Mathematik im Vordergrund (Gueudet 2008; Nagel und Reiss 2016). Studienanfängerinnen und -anfänger haben in der Schule für gewöhnlich Rückmeldungen zu ihren Leistungen in der Schulmathematik erhalten und darauf basierend ihr mathematisches Selbstkonzept entwickelt. An der Hochschule steht jedoch eine andere Art der Mathematik, die universitäre Mathematik, im Fokus der Lernprozesse. Es ist an dieser Stelle unklar, ob ein übergreifendes „mathematisches Selbstkonzept“ die Überzeugungen von angehenden Studierenden ausreichend differenziert beschreibt oder ob dieses zusätzlich nach den beiden Domänen - Schulmathematik und universitäre Mathematik - ausdifferenziert werden muss, um valide Aussagen über die Rolle des Selbstkonzepts in der Studieneingangsphase zu ermöglichen.

Der vorliegende Beitrag untersucht insgesamt, ob die Rolle des mathematischen Selbstkonzepts in der Studieneingangsphase mithilfe differenzierter Selbstkonzeptfacetten besser beschrieben werden kann. Dazu wird zunächst ein Fragebogeninstrument mit differenzierten Selbstkonzeptfacetten vorgestellt und dann mit einer Stichprobe von 202 Studierenden untersucht, ob sich die drei Facetten von Selbstkonzept - Selbstkonzept in Bezug auf die Mathematik, in Bezug auf die Schulmathematik und in Bezug auf die universitäre Mathematik - empirisch trennen lassen. Konkret wird anhand der Entwicklung des mathematischen Selbstkonzepts in der Studieneingangsphase der Mehrwert der differenzierten Facetten verdeutlicht. Basierend auf bekannten Modellen zur Genese des mathematischen Selbstkonzepts werden dazu Vermutungen zur Entwicklung aufgestellt und empirisch überprüft. Um Hinweise für die Validität des Instrumentes zu geben, werden erstens die Entwicklung der Selbstkonzeptfacetten von Studierenden unterschiedlicher Studiengänge (Fach Mathematik versus Lehramt Mathematik) miteinander verglichen und zweitens die prädiktive Kraft der Facetten entsprechend des Erlebens der Studieneingangsphase thematisiert.

\section{Mathematisches Selbstkonzept in der Studieneingangsphase}

\subsection{Kontext: Mathematische Lernprozesse in der Studieneingangsphase}

Unter einem Mathematikstudium verstehen wir im Folgenden ein Fachstudium Mathematik bzw. ein gymnasiales Lehramtsstudium mit Fach Mathematik. Als mögliche Ursachen für die geäußerten und gezeigten Schwierigkeiten von Studierenden (z.B. in Liebendörfer 2018) werden häufig zwei Veränderungen angeführt, die mit dem Übergang in ein Mathematikstudium einhergehen: eine Veränderung des Lernangebotes und dessen Nutzung sowie eine Veränderung der Domäne Mathematik (Gueudet 2008; Rach 2014). Die erste Veränderung ist in vielen Studiengängen zu konstatieren, da der didaktisch angeleitete Schulunterricht von Veranstaltungsformaten wie Vorlesung, Übung und Seminar abgelöst wird. Neben diesen Veranstal- 
tungsformaten bildet das Selbststudium den Kern des Studiums, für die der Hauptanteil an Lernzeit investiert wird (Wild 2005). In einem Mathematikstudium sind im Selbststudium häufig sehr anspruchsvolle mathematische Aufgaben zu bearbeiten (Liebendörfer und Göller 2016; Rach und Heinze 2013), was nach den Aussagen von Studierenden zu vielen Misserfolgssituationen führt (Liebendörfer 2018).

Die Veränderung des Lerngegenstandes ist für mathematische Studienprogramme besonders stark ausgeprägt, denn Mathematik als Schulfach und als Studienfach unterscheiden sich substanziell voneinander (Rach und Heinze 2017). Das Schulfach steht unter dem Ziel der Allgemeinbildung (Winter 1996). Aus diesem Grund spielen schematische Berechnungen und die Anwendung mathematischer Begriffe und Verfahren für außermathematische Problemstellungen in der Schulmathematik eine zentrale Rolle. Dagegen fokussiert das Studienfach auf die wissenschaftliche Disziplin Mathematik, die durch deduktive Beweise und formale Begriffsdefinitionen geprägt ist (Gueudet 2008; Nagel und Reiss 2016). Dieser Umbruch tritt sowohl in einem Fachbachelor- als auch in einem (gymnasialen) Lehramtsstudium auf. Diese beiden Arten von Mathematik differenzieren Studierende schon zu Beginn des ersten Semesters (vgl. Liebendörfer 2018; Rach et al. 2014; Ufer et al. 2017). Von Studierenden in einem Lehramtsstudium wird zudem berichtet, dass diese zur universitären Mathematik häufig keine tragfähige Beziehung aufbauen, da sie ihr wenig Relevanz für ihren späteren Beruf zuschreiben. Diese Studierenden berichten deshalb einen deutlichen Motivationsverlust im ersten Studienjahr (Hefendehl-Hebeker 2013; Liebendörfer 2018).

Da das Selbstkonzept ein domänenspezifisches (bereichsspezifisches) Konstrukt ist (Möller und Köller 2004), sind Ausdifferenzierungen des mathematischen Selbstkonzepts aufgrund einer anderen Art von Mathematik zu erwarten. Qualitative Studien in der Studieneingangsphase weisen darauf hin, dass Studierende ihre Einschätzungen und Erwartungen an den veränderten Lerngegenstand anpassen müssen, um erfolgreich zu sein. Diese Anpassungsprozesse gelingen vielen Studierenden jedoch nur mangelhaft (vgl. Daskalogianni und Simpson 2001; Liebendörfer 2018). Es stellt sich also die Frage, wie diese Anpassungsprozesse für das Konstrukt Selbstkonzept theoretisch beschrieben und empirisch zugänglich gemacht werden können.

\subsection{Definition und Struktur des mathematischen Selbstkonzepts}

Das Konstrukt Selbstkonzept wird als multidimensional und hierarchisch organisiert aufgefasst (Marsh et al. 1988; Möller und Köller 2004). Es lässt sich in eine akademische und eine nicht-akademische Facette unterteilen (Shavelson et al. 1976). Eine Unterfacette des akademischen Selbstkonzepts ist das allgemeine mathematische Selbstkonzept, das wir als die Einschätzung der eigenen mathematischen Fähigkeiten durch eine Person definieren (vgl. Bong und Skaalvik 2003). Diese Definition beschränkt sich auf die sogenannte evaluativ-kognitive Komponente von Selbstkonzept (,Ich bin fit in Mathematik.“) und umfasst nicht die emotionale Komponente (,Ich fühle mich wohl mit Mathematik.“) (Möller und Köller 2004). Aufgrund der Betonung der evaluativ-kognitiven Komponente sind Selbstkonzepte mit Selbstwirksamkeitserwartungen und Erfolgserwartungen verwandt: Eccles und Wigfield (2020) berichten von engen empirischen Zusammenhängen zwischen Erfolgserwartungen 
und Selbstkonzepten, plädieren aber aufgrund der unterschiedlichen theoretischen Konzeptualisierungen für eine Trennung der drei Konstrukte. Beispielsweise fokussieren Erfolgserwartungen und Selbstwirksamkeitserwartungen stärker die Einschätzung, wie erfolgreich eine spezifische Aufgabe oder eine Lernsituation in der Zukunft bewältigt wird (vgl. Bong und Skaalvik 2003; Marsh et al. 2019). Selbstkonzepte dagegen sind retrospektive Einschätzungen der eigenen Fähigkeiten, die auf schon erlebten Lernsituationen basieren.

\subsection{Genese und Entwicklung des mathematischen Selbstkonzepts}

Als wesentliche Informationsquellen für die Genese des Selbstkonzepts werden Vergleiche angenommen, auf denen die Bewertung der eigenen Fähigkeiten beruhen. Durch diese Vergleichsprozesse wird die Einschätzung der eigenen Fähigkeiten kalibriert. Im Internal/External Frame of Reference Modell (I/E-Modell, z. B. Marsh et al. 2015) werden dimensionale (internale) und soziale (externale) Vergleiche als zwei wichtige Vergleichsprozesse unterschieden (vgl. Möller und Köller 2004; Möller et al. 2009). Dimensionale Vergleiche betreffen Leistungen derselben Person in verschiedenen Domänen. Zwei Personen, die in Mathematik die gleichen Fähigkeiten aufweisen, können sich aufgrund dimensionaler Vergleiche in ihrem Selbstkonzept unterscheiden: Wenn Person A im Fach Englisch bessere Leistungen im Vergleich zum Fach Mathematik zeigt, Person B jedoch schlechtere Leistungen, so könnte Person A sich trotz gleicher mathematischer Fähigkeiten ein niedrigeres mathematisches Selbstkonzept zuschreiben als Person B. Bei sozialen Vergleichen schätzen Lernende ihre eigenen, mathematischen Fähigkeiten anhand von Mathematikleistungen anderer Lernender ein. In diesem Fall hängt die Anpassung des Selbstkonzepts von der Bezugsgruppe ab, anhand derer diese Vergleiche durchgeführt werden.

Weitere mögliche Vergleichsprozesse, welche die Entwicklung domänenspezifischer Selbstkonzepte beeinflussen können, sind temporale und kriteriale Vergleiche (Möller und Trautwein 2015; Skaalvik und Skaalvik 2002). Bei temporalen Vergleichen werden die individuellen Leistungen über die Zeit verglichen. Wenn sich die Leistungen der subjektiven Wahrnehmung nach verbessert haben und diese Verbesserung auf die eigenen Fähigkeiten attribuiert wird, dann wird ein Anstieg des Selbstkonzepts erwartet. Bei kriterialen Vergleichen werden die eigenen Leistungen anhand von selbst gesetzten oder übernommenen Zielkriterien bzw. Anforderungen bewertet. Erfüllen die individuellen Leistungen diese Kriterien, werden positive Auswirkungen auf das Selbstkonzept angenommen.

Bisherige Studien zur Entwicklung im Laufe der Schulzeit deuten darauf hin, dass das mathematische Selbstkonzept im Schulverlauf abnimmt (z. B. Fredricks und Eccles 2002; Nagy et al. 2010). Als Gründe für das Absinken im Laufe der Schulzeit werden beispielsweise eine realistischere Selbsteinschätzung sowie mehr und neue Vergleichsmöglichkeiten genannt (Jacobs et al. 2002). An institutionellen Übergängen konnte gezeigt werden, dass der Übergang auf eine weiterführende Schule eines höheren Anspruchsniveaus mit einem sinkenden mathematischen Selbstkonzept einhergeht (Becker und Neumann 2016; Wigfield et al. 1991). 


\subsection{Struktur und Entwicklung des mathematischen Selbstkonzepts in der Studieneingangsphase}

Eine Ausdifferenzierung des mathematischen Selbstkonzepts in der Studieneingangsphase erscheint gerechtfertigt, wenn sie theoretisch begründet wird, die postulierten Facetten sich als empirisch trennbar erweisen und die Facetten differenzierte Aussagen beispielsweise über die Entwicklung des mathematischen Selbstkonzepts erlauben. Es kann angenommen werden, dass den Studierenden zu Beginn des Studiums viele Informationen zu ihren schulischen Mathematikleistungen vorliegen, jedoch nur wenige Informationen zu ihren (möglichen) Leistungen in Bezug auf die universitäre Mathematik. Um ein spezifisches Selbstkonzept zur universitären Mathematik aufzubauen, sind soziale, dimensionale und temporale Vergleiche damit kaum möglich. Es gibt Hinweise in der Literatur, dass Studienanfängerinnen und -anfänger wichtige Anforderungen eines Mathematikstudiums kennen (Rach et al. 2014), was kriteriale Vergleiche erlauben könnte. Die potenzielle Bewältigung dieser Anforderungen würde jedoch auf der Basis bisheriger (schulischer) Leistungen bewertet. Damit erscheint es fraglich, ob schon zu Beginn der Studieneingangsphase eine trennbare Selbstkonzeptfacette zur universitären Mathematik erfasst werden kann.

Je nach vorherrschendem Vergleichsprozess werden unterschiedliche Entwicklungen des mathematischen Selbstkonzepts in der Studieneingangsphase erwartet. Der Übergang in eine neue Lerngruppe verändert den Kontext für soziale Vergleiche. Es ist plausibel, dass vor allem Schulabsolventinnen und -absolventen mit besseren mathematischen Leistungen und einem höheren mathematischen Selbstkonzept ein mathematikintensives Studium aufnehmen (theoretisch begründet durch ErwartungsWert-Modelle z.B. in Eccles und Wigfield 2020; empirisch untermauert z. B. durch Parker et al. 2012 und Wang 2013). Somit sind die Leistungen der Referenzgruppe für soziale Vergleiche in der Studieneingangsphase höher als im Schulkontext, was zu einem Rückgang aller Selbstkonzeptfacetten führen kann (s.a. Big-Fish-LittlePond-Effekt, Marsh 2005).

Der in der Literatur beschriebene und von Studierenden auch wahrgenommene Unterschied zwischen Schul- und universitärer Mathematik (z.B. Gueudet 2008; Rach et al. 2014) ermöglicht zusätzliche dimensionale Vergleiche, indem zwischen den beiden Arten von Mathematik verglichen wird. Dimensionale Vergleiche sind auch in der Schule zu finden, dort jedoch häufig zwischen Fächern. Da in einem Studium das Fächerspektrum im Vergleich zur Oberstufe deutlich eingeschränkt ist, erscheinen dimensionale Vergleiche innerhalb eines Faches, beispielsweise zwischen verschiedenen Arten von Mathematik, als plausibel. In der Literatur wird oftmals auf die hohen Anforderungen verwiesen, die beim Lernen von universitärer Mathematik insbesondere in der Studieneingangsphase zu bewältigen sind und die von den Studierenden z. T. nicht bewältigt werden (Liebendörfer 2018; Rach und Heinze 2013). Durch diese nicht bewältigten Anforderungen könnten sich die Studierenden als weniger leistungsstark in Bezug auf die universitäre Mathematik einschätzen, als sie sich in Bezug auf die Schulmathematik eingeschätzt haben. Wenn nun die Studierenden ihre Leistungen entsprechend der Arten von Mathematik miteinander vergleichen, kann erwartet werden, dass dieser dimensionale Vergleich zu einer ne- 
gativen Kalibrierung des Selbstkonzepts zur universitären Mathematik und zu einer positiven Kalibrierung des Selbstkonzepts zur Schulmathematik führt. Erste empirische Hinweise auf ähnliche dimensionale Vergleiche im Lehramtsstudium haben beispielsweise Sorge et al. (2019) berichtet: Bei Lehramtsstudierenden im Fach Physik hängt das fachliche Wissen unter Kontrolle von fachdidaktischem Wissen negativ mit dem fachdidaktischen Selbstkonzept zusammen.

Die z. T. nicht bewältigten fachlichen Anforderungen in der Studieneingangsphase erscheinen auch relevant für kriteriale Vergleiche, die in Bezug auf die universitäre Mathematik von niedrigen Leistungen geprägt sein dürften. Auf eine entsprechende Selbstkonzeptfacette sollten sich die z. T. nicht bewältigten Anforderungen nur dann negativ auswirken, wenn die Lernenden lediglich ihre wahrgenommenen oder die ihnen rückgemeldeten Leistungen für die Kalibrierung heranziehen und nicht das höhere Niveau der dahinterstehenden Anforderungen berücksichtigen. Da für Leistungen zur Schulmathematik kaum neue Informationen anfallen, könnten kriteriale Vergleiche kaum als Erklärung für eine eventuelle Veränderung des schulischen mathematischen Selbstkonzepts dienen.

Ähnliches gilt in Bezug auf temporale Vergleiche. Ein Anstieg des allgemeinen mathematischen Selbstkonzepts wäre plausibel, wenn die Studierenden auch Teilerfolge bei Anforderungen höherer Komplexität positiv bewerten. Ein Rückgang des allgemeinen mathematischen Selbstkonzepts wäre jedoch auch denkbar, wenn die Studierenden nur die zunehmenden fachlichen Schwierigkeiten und ihre schlechteren Leistungen im zeitlichen Verlauf zur Kalibrierung ihres Selbstkonzepts heranziehen.

Studierendenbefragungen im ersten Semester deuten darauf hin, dass Studierende häufig kriteriale Vergleiche anstellen, um ihre eigene Kompetenz einzuschätzen (Liebendörfer 2018). Diese Beobachtung ist ggf. dadurch zu erklären, dass Studierende aufgrund erhöhter Anforderungen mehr Misserfolge erleben und die Studierenden diese Misserfolge dann verwenden, um ihr universitäres mathematisches Selbstkonzept zu kalibrieren. Dass Studierende soziale und temporale Vergleiche verwenden, wird dagegen deutlich seltener berichtet. Zusammenfassend legen unterschiedliche Vergleichsmöglichkeiten eine differentielle Entwicklung von Selbstkonzeptfacetten nahe. Dies lässt eine Ausdifferenzierung des individuellen mathematischen Selbstkonzepts von Mathematikstudierenden in ein universitäres, ein schulisches und ein allgemeines mathematisches Selbstkonzept im ersten Semester als plausibel erscheinen.

\subsection{Mathematisches Selbstkonzept und das Erleben der Studieneingangsphase}

Dass viele Studierende ihr Studium gerade zu Beginn wieder abbrechen (Dieter 2012), wird vor allem auf zwei Faktoren zurückgeführt: Schwierigkeiten beim Fachwissenserwerb und mangelnde Studienmotivation (Heublein et al. 2009; Neugebauer et al. 2019). Das Konstrukt Selbstkonzept wird als ein Frühindikator eines Studienabbruches in der Literatur zwar diskutiert, ist aber nicht hinreichend untersucht aktuelle Ergebnisse sind beispielsweise in Geisler (2020) zu finden. Schiefele et al. (2007) berichten keine signifikanten Unterschiede im akademischen Selbstkonzept zwischen Weiterstudierenden und Studienabbrechenden sowohl zu Studienbeginn als auch zum Zeitpunkt des Studienabbruches. Dabei bestand ihre Stichprobe allerdings 
aus Studierenden verschiedener Fachrichtungen. In dieser Studie zur Vorhersage eines Studienabbruches zeigt sich jedoch, dass Studienabbrecherinnen und -abbrecher eine höhere Demotivation und eine deutlich niedrigere Studienzufriedenheit als Weiterstudierende berichteten. Somit steht das Phänomen des Studienabbruches mit dem Erleben der Studieneingangsphase in Verbindung.

Qualitative Analysen liefern Hinweise auf die Wirkweise des Selbstkonzepts auf den Studienabbruch, speziell in einem Mathematikstudium (Bampili et al. 2017; Di Martino und Gregorio 2019). Dabei wird vermutet, dass insbesondere eine negative Entwicklung des Selbstkonzepts in der Studieneingangsphase einen Studienabbruch prädiziert. Weiterhin werden mit dieser Anpassung verbundene negative Emotionen, Demotivation und geringe Studienzufriedenheit als Vermittler für eine erhöhte Studienabbruchtendenz und, im weiteren Verlauf, für einen Studienabbruch diskutiert (Di Martino und Gregorio 2019; Fleischer et al. 2019). Diese Variablen, die das individuelle Erleben der Studieneingangsphase beschreiben, werden also als wesentliche Vermittler zwischen individuellen Studienvoraussetzungen und einem konkreten Studienabbruch angenommen. Entsprechend ist zu vermuten, dass mathematisches Selbstkonzept als Frühindikator für ein positives Erleben der Studieneingangsphase und damit für eine reduzierte Tendenz zum Studienabbruch dienen kann.

Das Erleben der Studieneingangsphase kann durch verschiedene Maße bestimmt werden: Das Konstrukt Studienzufriedenheit beschreibt die studentische Beurteilung des eigenen Studienganges und basiert beispielsweise auf der Bewertung, inwieweit individuelle Erwartungen und aktuelle Studienerfahrungen zueinander passen (Blüthmann 2012). Die Konstrukte Motivation bzw. Demotivation sind Indikatoren, die die Bereitschaft in den Blick nehmen, sich mit den Lerninhalten verstärkt oder eben nicht auseinander zu setzen. Die Stabilität der Studienwahl fokussiert die retrospektive Beurteilung der Studienwahl, also inwiefern der gewählte Studiengang bei einer erneuten Entscheidung wiedergewählt werden würde (Brandstätter et al. 2002). Für den Zusammenhang des akademischen Selbstkonzepts mit der Studienzufriedenheit bzw. der Studienmotivation sind uns nur wenige Studien bekannt. Als wichtiger Prädiktor für die Studienzufriedenheit wird das domänenspezifische Interesse und weniger das fachliche Vorwissen angesehen (z. B. Blüthmann 2012; Kosiol et al. 2019; Schiefele und Jacob-Ebbinghaus 2006). Speziell für Lehramtsstudierende gibt es aber auch Hinweise auf positive Zusammenhänge von akademischem Selbstkonzept und der Zufriedenheit mit Studieninhalten (Bernholt et al. 2018) sowie geringerer Prokrastination (Lohbeck et al. 2017). Aufgrund des Zusammenspiels des Selbstkonzepts mit der Fachleistung und der Studienzufriedenheit kann angenommen werden, dass ein hohes Selbstkonzept zur Stabilität der Wahl des anspruchsvollen Mathematikstudiums beiträgt. Die Annahme, dass Lernende mit einem höheren mathematischen Selbstkonzept überhaupt eher anspruchsvollere Kurse wählen (und diese dann auch weiterführen), kann durch die Ergebnisse von Köller et al. (2006) gestützt werden. 


\section{Untersuchung zur Struktur und Entwicklung des mathematischen Selbstkonzepts in der Studieneingangsphase}

Insgesamt lassen sich aus dem Forschungsstand die folgenden Annahmen ableiten: (1) Die Domäne Mathematik ändert sich beim Übergang in ein Mathematikstudium.

(2) Es ist plausibel, dass diese Änderung zu einer Ausdifferenzierung des mathematischen Selbstkonzepts führen kann. (3) Aufgrund der Bedeutung des domänenspezifischen Selbstkonzepts für Lernprozesse ist es von Interesse, inwiefern spezifische Selbstkonzeptfacetten einen Mehrwert bieten, die Entwicklung des mathematischen Selbstkonzepts zu beschreiben. (4) Um die Validität der differenzierten Messung zu stützen, kann die prädiktive Validität der Skalen in Bezug auf das Erleben der Studieneingangsphase betrachtet werden.

Ausgehend von diesen Annahmen wurde ein Fragebogeninstrument entwickelt, das eine allgemeine mathematische Facette sowie eine auf die schulische und eine auf die universitäre Mathematik bezogene Facette des mathematischen Selbstkonzepts erfasst. Um die Struktur, Entwicklung und Prädiktionskraft dieser Selbstkonzeptfacetten zu untersuchen, wurde das Instrument bei $N=202$ Studienanfängerinnen und Studienanfängern zu Beginn sowie in der Mitte des ersten Semesters eingesetzt. Dabei sind die folgenden vier Fragestellungen zentral:

1. Lassen sich eine allgemeine, eine auf die schulische und eine auf die universitäre Mathematik bezogene Facette des mathematischen Selbstkonzepts zu Beginn eines Mathematikstudiums bzw. in der Mitte des ersten Semesters empirisch trennen?

Aufgrund der geringen individuellen Vergleichsmöglichkeiten in Bezug aufdie universitäre Mathematik erwarten wir keine Trennbarkeit der drei Facetten zu Studienbeginn. Wir erwarten jedoch, dass die zusätzlichen Möglichkeiten für soziale, dimensionale, temporale und kriteriale Vergleiche im Verlauf des Semesters zu einer Ausdifferenzierung der Facetten führen. Demnach erwarten wir, dass ein eindimensionales Modell zu Studienbeginn die Daten besser repräsentiert, während zur Mitte des ersten Semesters ein dreidimensionales Modell besser auf die Daten passt.

2. Wie entwickeln sich die verschiedenen Facetten des mathematischen Selbstkonzepts zu Beginn eines Mathematikstudiums?

Wir vermuten, dass das allgemeine mathematische Selbstkonzept infolge sozialer Vergleiche im Verlauf des ersten Semesters eines Mathematikstudiums abnimmt (Geisler 2020; Rach und Heinze 2013). Falls sich die Facetten schon zu Studienbeginn trennen lassen, erwarten wir zudem, dass aufgrund der erhöhten Anforderungen in der neuen Domäne der universitären Mathematik die universitäre Facette durch temporale und kriteriale Vergleichsprozesse abnimmt. Veränderungen der schulischen Facette erwarten wir nicht, da die Schulmathematik nicht Lerngegenstand ist und somit keine neuen Vergleichsmöglichkeiten eröffnet werden.

3. Inwiefern unterscheidet sich die Entwicklung der Selbstkonzeptfacetten zwischen Studierenden in einem Fach- versus in einem gymnasialen Lehramtsstudium?

Angesichts kriterialer Vergleiche erwarten wir eine stärkere Abnahme des universitären mathematischen Selbstkonzepts bei Lehramtsstudierenden, da diese ihr 
Studium im Mittel mit geringeren Vorkenntnissen als Fachstudierende beginnen (Rach 2014). Für das allgemeine mathematische Selbstkonzept erwarten wir keine Interaktion mit dem Studiengang, da soziale Vergleichsprozesse unabhängig vom gewählten Studiengang ablaufen.

4. Inwiefern sagen die Selbstkonzeptfacetten Maße des Studienerlebens, z. B. Demotivation, Stabilität der Studienwahl und Studienzufriedenheit, in der Mitte des ersten Semesters voraus?

Wir erwarten, dass das mathematische Selbstkonzept das Erleben vorhersagt: Da das Selbstkonzept auf der Einschätzung der eigenen Fähigkeiten beruht, hängt ein hohes Selbstkonzept sicherlich mit einer erfolgreichen Bewältigung von Anforderungen zusammen. Eine erfolgreiche Bewältigung der Anforderungen sollte die Studierenden weniger demotivieren, ihre Studienzufriedenheit erhöhen und somit zur Stabilität der Studienwahl beitragen. Diese Zusammenhänge erwarten wir insbesondere für das universitäre mathematische Selbstkonzept.

\section{Methodisches Vorgehen}

Die berichtete Studie ist im Projekt SISMa (Selbstkonzept und Interesse in der Studieneingangsphase Mathematik) angesiedelt. In diesem Beitrag fokussieren wir das Konstrukt Selbstkonzept - Ergebnisse zum Konstrukt Interesse sind in Ufer et al. (2017) und Kosiol et al. (2019) publiziert.

\subsection{Stichprobe und Design}

Studierende des ersten Studiensemesters Mathematik einer Universität einer deutschen Großstadt wurden in der ersten Mathematikvorlesung zur Teilnahme rekrutiert (T1) und erneut nach acht Wochen (T2) befragt. Es liegen verwertbare Daten von 352 Studierenden vor, wobei 103 Datensätze keine Daten zu T2 enthalten und 47 Datensätze keine Daten zu T1. In die Analysen sind Daten der verbleibenden $N=202$ Studierenden (95 weiblich) eingeflossen (vgl. Kosiol et al. 2019), die an zwei Befragungszeitpunkten teilnahmen. Die fehlenden Daten zum zweiten Messzeitpunkt könnten teilweise auf einen sehr frühen Studienabbruch von Studierenden zurückzuführen sein (vgl. Geisler 2020), die ihr Studium nicht ernsthaft aufgenommen haben. Datensätze mit fehlenden Daten zum ersten Messzeitpunkt konnten gerade für die Fragen zur Entwicklung der Struktur der Selbstkonzeptfacetten für diese Studie nicht berücksichtigt werden. Eingeschrieben waren $n=119$ Studierende (45\% weiblich; Alter: $M=19,2, S D=1,8)$ im Fachbachelor Mathematik bzw. Fachbachelor Wirtschaftsmathematik, und $n=74$ Studierende (55\% weiblich; Alter: $M=19,7, S D=1,9)$ in einem gymnasialen Lehramtsstudiengang mit Fach Mathematik. Zu neun Studierenden lag keine Angabe zum Studiengang vor. Studierende der Bachelor-Studiengänge (Wirtschafts-)Mathematik nahmen an derselben mathematischen Lehrveranstaltung „Analysis I“ im ersten Semester teil, Lehramtsstudierende besuchten eine eigene mathematische Lehrveranstaltung „Analysis I“ mit vergleichbaren Inhalten. Beide Lehrveranstaltungen fokussierten Mathematik als deduktive Wissenschaft mit formalen Begriffsdefinitionen und deduktiven Beweisen. Bei 
Tab. 1 Verwendete Skalen für mathematische Selbstkonzeptfacetten mit Beispielitems

\begin{tabular}{|c|c|c|}
\hline Skalen & Anzahl Items & Beispielitem \\
\hline Allgemein & 4 & „Ich denke, Mathematik liegt mir besonders.“ \\
\hline Schulisch & 3 & $\begin{array}{l}\text { „Was die Mathematik angeht, die ich in der Schule kennengelernt } \\
\text { habe, bin ich ziemlich fit.“ }\end{array}$ \\
\hline Universitär & 3 & $\begin{array}{l}\text { „Die Mathematik, die an der Hochschule betrieben wird, fällt mir } \\
\text { leicht.“ }\end{array}$ \\
\hline
\end{tabular}

dieser Stichprobe handelt es sich um eine Gelegenheitsstichprobe, die einer gesamten Kohorte der Studiengänge Mathematik, Wirtschaftsmathematik und gymnasiales Lehramt mit Fach Mathematik einer Universität entstammt. Die Studierenden wurden über das Projekt in den Vorlesungen informiert und nahmen freiwillig an der Befragung teil.

Die Studierenden füllten zu beiden Messzeitpunkten einen Fragebogen zu den drei mathematischen Selbstkonzeptfacetten aus. Drei Maße für das Studienerleben wurden ebenfalls zur Semestermitte (T2) erhoben. Die Studierenden berichteten ihre Studienzufriedenheit mit dem Mathematikstudium (7 Items, $\alpha=0,83, N=202$, Beispielitem: „Insgesamt bin ich mit meinem Mathematikstudium zufrieden.“, vgl. Schiefele und Jacob-Ebbinghaus 2006) und ihre Demotivation (6 Items, $\alpha=0,85$, $N=201$, Beispielitem: „Ich frage mich oft, was ich hier im Mathematikstudium eigentlich soll.“, vgl. Schiefele et al. 2002). Die Aussagen zu allen Skalen wurden auf einer vierstufigen Likert-Skala von ,trifft zu“ (3) bis „,trifft nicht zu“ (0) eingeschätzt. Außerdem haben die Studierenden angegeben, wie stabil ihre Studienwahl ist (2 Items, $r=0,69, N=202$, Beispielitem: „Würden Sie Ihr gegenwärtiges Studium noch einmal wählen, wenn Sie sich noch einmal entscheiden müssten?“, Antwortalternativen: ,ja, ganz bestimmt“ (3), ,wahrscheinlich“ (2), ,wahrscheinlich nicht“(1), „bestimmt nicht“(0), vgl. Brandstätter et al. 2002).

\subsection{Instrumente zur Messung der Selbstkonzeptfacetten}

Tab. 1 gibt einen Überblick über die verwendeten Skalen für die Selbstkonzeptfacetten. Das allgemeine mathematische Selbstkonzept wurde mit Hilfe der etablierten Skala aus dem PaLea-Projekt (Kauper et al. 2012) erhoben. Die spezifischen Selbstkonzeptskalen wurden im Projekt SISMa neu entwickelt. Bis auf die Referenz der unterschiedlichen Institutionen Schule bzw. Hochschule stimmen die Itemformulierungen dieser beiden Skalen überein - alle Items der neu entwickelten Skalen sind in Tab. 8 im Anhang dargestellt. Zu T1 wurden die Studierenden darauf hingewiesen, die Items zur Institution Universität so zu beantworten, wie sie sich die universitäre Mathematik vorstellten. Insgesamt fehlen auf Ebene der Einzelitems 3,4\% der Daten.

\section{Ergebnisse}

Dieser Beitrag widmet sich der Frage, ob differenzierte Selbstkonzeptfacetten dazu beitragen, die Rolle des mathematischen Selbstkonzepts für universitäre Lernprozes- 
se differenzierter zu beschreiben als ein einziges mathematische Selbstkonzeptmaß. In diesem Teil werden zuerst Ergebnisse zur Struktur des mathematischen Selbstkonzepts beschrieben und anhand der Entwicklung des mathematischen Selbstkonzepts aufgezeigt, welche Einblicke in Entwicklungsprozesse die differenzierten Messungen ermöglichen. Zur Validierung des Instrumentes werden zweierlei Analysen genutzt: Erstens werden Unterschiede im mathematischen Selbstkonzept zwischen Studiengängen berichtet und zweitens wird fokussiert, wie die Selbstkonzeptfacetten mit dem Erleben der Studieneingangsphase zusammenhängen.

\subsection{Struktur des Selbstkonzepts}

Analysestrategie: Zur Trennbarkeit der Selbstkonzeptfacetten werden drei Modelle miteinander verglichen: Modell 1 geht von einem eindimensionalen Selbstkonzeptkonstrukt zu beiden Messzeitpunkten aus, Modell 2 von einem eindimensionalen Konstrukt zu T1 und einem dreidimensionalen Konstrukt zu T2 und Modell 3 von einem dreidimensionalen Konstrukt zu beiden Messzeitpunkten (siehe Abb. 1). Aufgrund der wenigen Vergleichsmöglichkeiten in Bezug auf die universitäre Mathematik zu Studienbeginn sind wir davon ausgegangen, dass Modell 2 die Daten am besten modelliert. Die Modelle wurden mit Hilfe einer konfirmatorischen Faktorenanalyse mit dem Paket ,lavaan“ in R geschätzt.

Beide Modelle mit eindimensionalen Konstrukten weisen keinen akzeptablen Fit auf (Tab. 2). Die Fähigkeitsselbsteinschätzungen der Studierenden lassen sich nicht zu beiden Messzeitpunkten mit einem allgemeinen mathematischen Selbstkonzept erklären. Nur Modell 3 mit zwei dreidimensionalen Konstrukten zeigt einen akzeptablen Fit, und es beschreibt die Daten signifikant besser als Modell 2. Entgegen unserer Erwartung lässt sich somit schon zu Beginn des Studiums eine dreidimensionale Struktur identifizieren, die nach acht Wochen weiterhin Bestand hat. ${ }^{1}$

Um sicherzustellen, dass das Instrument invariant über die Zeit misst, wurde Modell 3 aus Tab. 2 weiter restringiert. Das finale Modell ist in Abb. 2 dargestellt und weist die folgenden Modellkriterien auf: $\chi^{2}(164)=278,68, C F I=0,929$, RMSEA $=0,060$. In diesem Modell sind bis auf die Ladung beim Item Sk_A2 alle Faktorladungen längsschnittlich restringiert.

Die deskriptiven Daten der drei Skalen sowohl zu Beginn als auch in der Mitte des ersten Semesters sind in Tab. 3 dargestellt. Die Reliabilitäten der Skalen sind insgesamt als zufriedenstellend bis gut zu bewerten, in der Skala ,schulisches mathematisches Selbstkonzept“" sind leichte Deckeneffekte zu erkennen. Die manifesten Korrelationen zwischen dem allgemeinen und dem universitären mathematischen Selbstkonzept sind zu beiden Zeitpunkten hoch, die Korrelation zwischen dem allgemeinen und dem schulischen mathematischen Selbstkonzept ist zu Beginn hoch und liegt in der Mitte des ersten Semesters auf mittlerem Niveau (vgl. Tab. 5 im

\footnotetext{
${ }^{1}$ Ebenfalls getrennt für die beiden Studiengänge Fach und Lehramt Mathematik sind die drei Modelle gerechnet worden. Es zeigt sich, dass das Modell 3 gegenüber Modell 1 und 2 in beiden Studienganggruppen überlegen war, wobei die Modellindizes für die kleinen Stichprobengröße noch akzeptabel sind: Fach, $n=119, \chi^{2}(155)=263,50, \mathrm{CFI}=0,893$, RMSEA $=0,077$, Lehramt, $n=74, \chi^{2}(155)=257,74, \mathrm{CFI}=0,837$, RMSEA $=0,095$. Aufgrund der kleinen Stichprobengrößen haben wir keine weiteren Invarianzanalysen für die Studiengänge durchgeführt.
} 

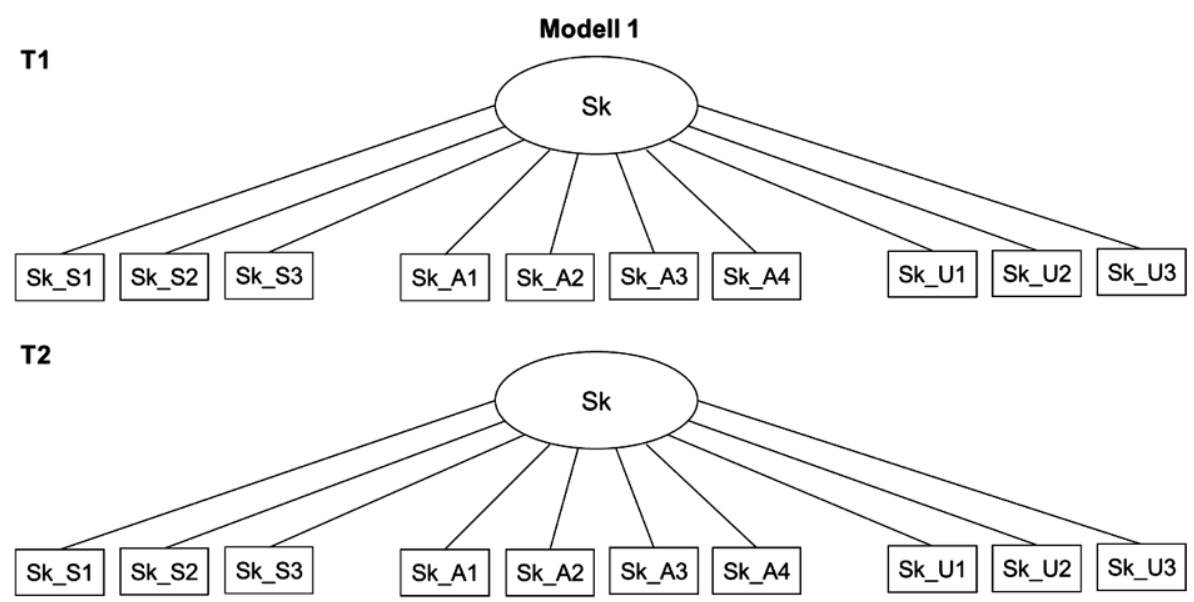

Modell 2
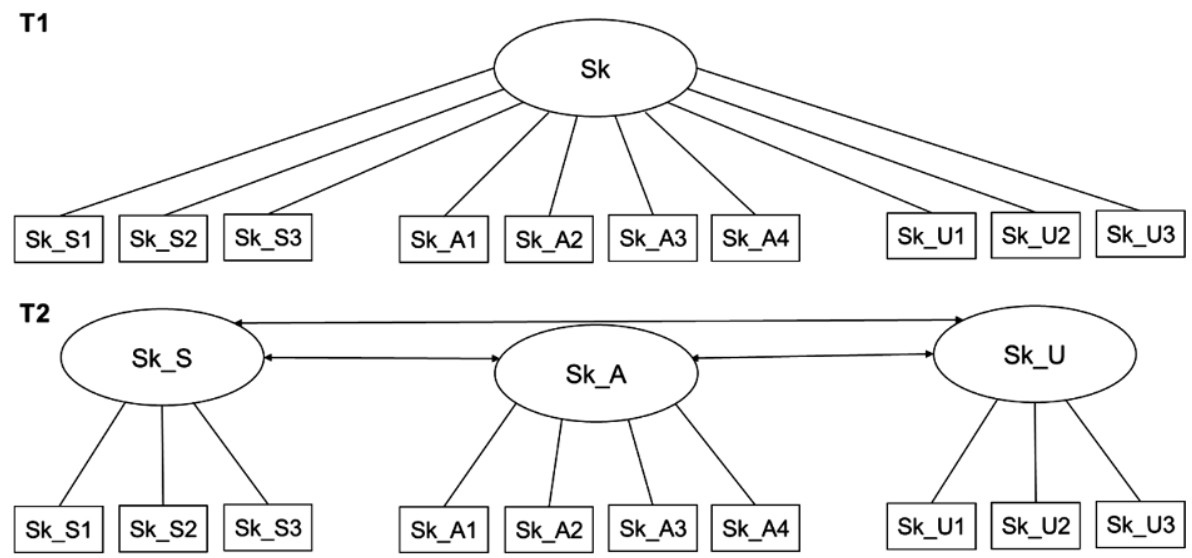

Modell 3
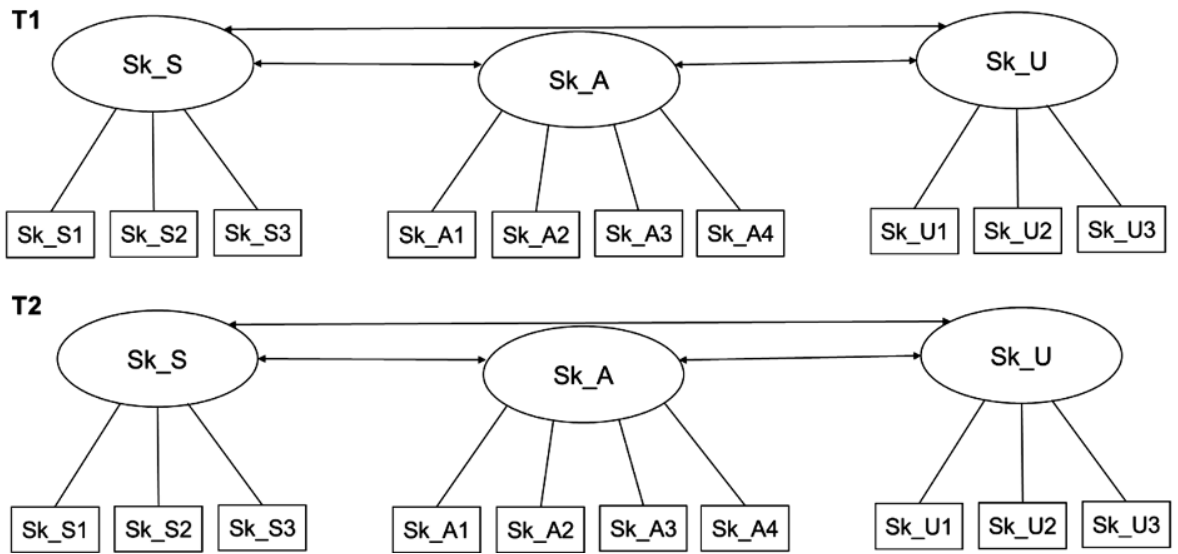

Abb. 1 Drei theoretische Modelle zur Struktur des Selbstkonzepts. ( $S k \_A$ allgemeines math. Selbstkonzept, $S k \_U$ universitäres math. Selbstkonzept, $S k \_S$ schulisches math. Selbstkonzept) 
Tab. 2 Modellvergleich zur Struktur des mathematischen Selbstkonzepts

\begin{tabular}{lllllll}
\hline Modell & T1 & T2 & CFI & RMSEA & $\chi^{2}$ & $\Delta \chi^{2}$ \\
\hline 1 & 1D & 1D & 0,551 & 0,150 & $\chi^{2}(169)=910,1$ & - \\
2 & 1D & 3D & 0,761 & 0,111 & $\chi^{2}(164)=551,5$ & $\Delta \chi^{2}(5)=1055,4^{* * *}$ \\
3 & 3D & 3D & 0,932 & 0,061 & $\chi^{2}(155)=265,0$ & $\Delta \chi^{2}(9)=310,4^{* * *}$ \\
\hline
\end{tabular}

$N=202$

T1 Beginn des Studiums, T2 Nach acht Wochen $* * * p<0,001$

Tab. 3 Skalen für mathematische Selbstkonzeptfacetten mit Mittelwerten $(M)$, Standardabweichungen $(S D)$ und Cronbachs Alpha $(\alpha)$ zu Beginn (T1) und zur Mitte (T2) des ersten Semesters

\begin{tabular}{lllll}
\hline Skalen & T1 & \multicolumn{3}{c}{ T2 } \\
& $M(S D)$ & $\alpha$ & $M(S D)$ & $\alpha$ \\
\hline Allgemein $(N=202$ bzw. $N=201)$ & $2,2(0,46)$ & 0,75 & $1,7(0,57)$ & 0,84 \\
Schulisch $(N=195$ bzw. $N=197)$ & $2,4(0,51)$ & 0,69 & $2,5(0,48)$ & 0,69 \\
Universitär $(N=164$ bzw. $N=193)$ & $1,6(0,65)$ & 0,87 & $1,1(0,68)$ & 0,87 \\
\hline
\end{tabular}

Vierstufige Likert-Skala von „,trifft zu“ (3) bis ,trifft nicht zu“ $(0)$

Anhang). Es gibt keine Hinweise, dass das universitäre mathematische Selbstkonzept und das schulische mathematische Selbstkonzept miteinander zusammenhängen. Insgesamt scheinen sich das schulische mathematische und das allgemeine mathematische Selbstkonzept in den ersten Studienwochen voneinander zu entkoppeln.
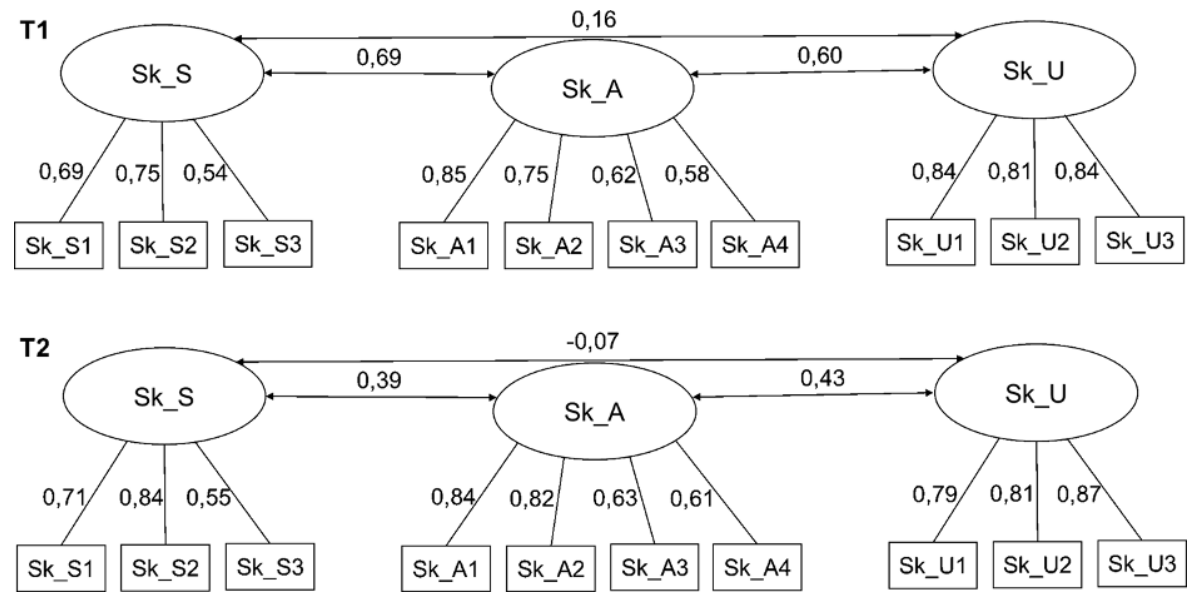

Abb. 2 Finales Modell mit standardisierten Faktorladungen und Korrelationen zwischen den latenten Merkmalen. Alle Faktorladungen und Korrelationen (Ausnahme: Korrelation zwischen universitärem und schulischem Selbstkonzept) sind auf dem 0,001-Niveau signifikant von Null verschieden. (Sk_A allgemeines math. Selbstkonzept, $S k \_U$ universitäres math. Selbstkonzept, $S k \_S$ schulisches math. Selbstkonzept) 


\subsection{Entwicklung der Selbstkonzeptfacetten}

Die Entwicklung der differenzierten Selbstkonzeptfacetten wird mittels Varianzanalysen mit Messwiederholung und mit dem Faktor Studiengang (für die Fragestellung 3; R-Paket „lme4“) analysiert. Abb. 3 stellt die Entwicklungen der Selbstkonzeptfacetten in den ersten acht Semesterwochen dar. Während das schulische Selbstkonzept sich erwartungsgemäß im Mittel nicht verändert $(F(1,189)=2,09$, $\left.p=0,15, \eta^{2}=0,01\right)$, nimmt sowohl das allgemeine mathematische Selbstkonzept $\left(F(1,193)=162,70, p<0,001, \eta^{2}=39\right)$ als auch das universitäre Selbstkonzept $\left(F(1,173)=86,41, p<0,001, \eta^{2}=0,26\right)$ mit starkem Effekt ab.

\subsection{Entwicklung der Selbstkonzeptfacetten im Zusammenhang mit dem Studiengang}

Bachelorstudierende berichten im Mittel ein höheres universitäres mathematisches Selbstkonzept als Lehramtsstudierende. Dieser Haupteffekt des Faktors Studiengang ist nur beim universitären Selbstkonzept nachweisbar: $F(1,195)=20,00, p<0,001$. Im Hinblick auf die Entwicklungen der Facetten gelten für beide Studienganggruppen ähnliche Muster (vgl. Abschn. 5.2), wobei jedoch ein signifikanter Interaktionseffekt zwischen Zeit und Studiengang für die universitäre Facette auftritt $\left(F(1,173)=4,69, p<0,05, \eta^{2}=0,01\right)$ : Das universitäre mathematische Selbstkonzept der Lehramtsstudierenden nimmt im Laufe des ersten Semesters stärker ab als das der Bachelorstudierenden (siehe Abb. 3). Diese unterschiedlichen Entwicklungsverläufe hatten wir erwartet, da Lehramtsstudierende ihr Studium mit im Mittel geringeren Vorkenntnissen als Fachstudierende beginnen. Weitere Interaktionen zeigen sich nicht.

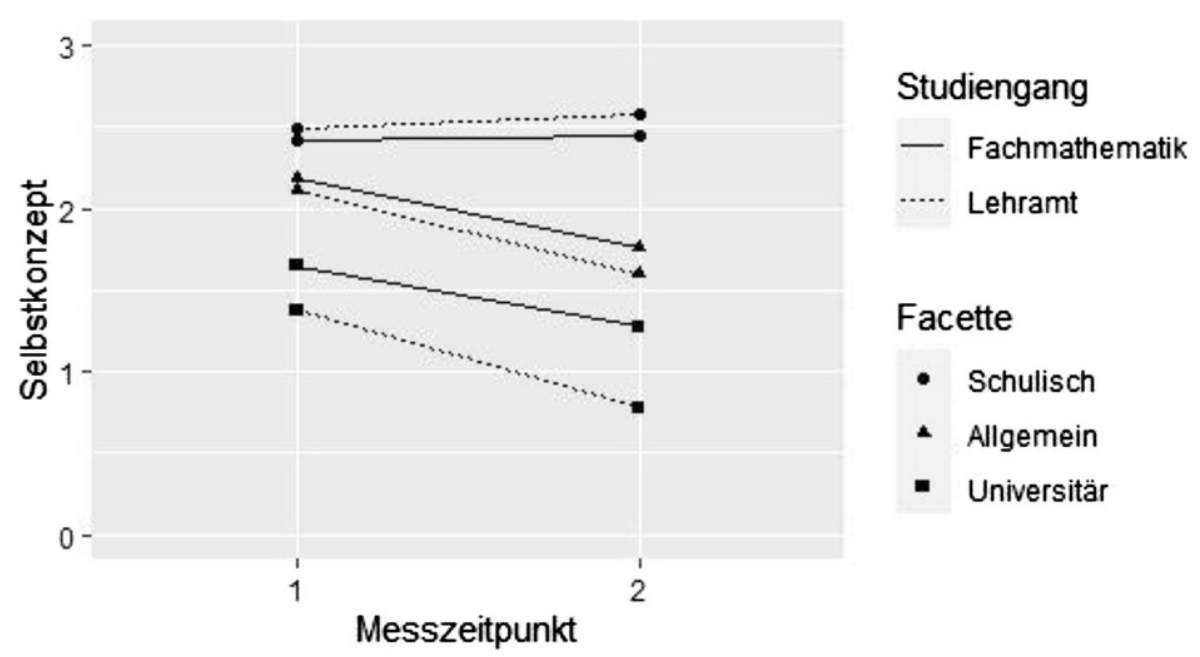

Abb. 3 Entwicklung der Selbstkonzeptfacetten vom Beginn (1) bis zur Mitte des ersten Semesters (2);

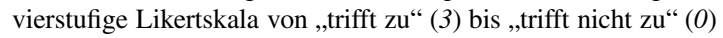




\subsection{Prädiktion des Erlebens der Studieneingangsphase durch die Selbstkonzeptfacetten}

Um die Validität der neuen Skalen noch besser einschätzen zu können, wird die prädiktive Validität dieser in Bezug auf das Erleben der Studieneingangsphase untersucht. Mittels Regressionsanalysen mit dem Paket „lavaan“ in R (fehlende Daten mit FIML geschätzt) wird analysiert, welche Selbstkonzeptfacetten die drei Maße des Erlebens der Studieneingangsphase (Studienzufriedenheit, Demotivation, Stabilität der Studienwahl) prädizieren. Insgesamt werden jeweils fünf Modelle für jedes Maß spezifiziert (siehe Tab. 4 für die Studienzufriedenheit; sowie Tab. 6 im Anhang für die Demotivation und Tab. 7 im Anhang für die Stabilität), wobei zunächst nur der Studiengang und dann getrennt nach Messzeitpunkt zuerst das allgemeine mathematische Selbstkonzept und dann die differenzierten Selbstkonzeptfacetten aufgenommen werden. Auffällig ist bei allen drei Maßen, dass der Studiengang allein kaum identifizierbare Varianz aufklärt (jeweils Modell 1), wobei Bachelorstudierende die Studieneingangsphase noch leicht positiver erleben als Lehramtsstudierende. Konform mit unseren Erwartungen spielt das Selbstkonzept eine größere Rolle für das Studienerleben. Dabei sagen primär die Selbstkonzeptfacetten zur Mitte des ersten Semesters das Erleben der Studieneingangsphase vorher und weniger die Selbstkonzeptfacetten zu Beginn (Modelle 2 und 4 bzw. Modelle 3 und 5). Insbesondere die Zufriedenheit und die Demotivation können durch differenzierte Selbstkonzeptfacetten besser vorhergesagt werden als durch das allgemeine mathematische Selbstkonzept allein (Modelle 2 und 3 bzw. Modelle 4 und 5). Wie erwartet, hängt dabei das universitäre Selbstkonzept mit einem positiven Erleben zusammen. Für das schulische Selbstkonzept sind keine signifikanten Zusammenhänge nachweisbar. Auffällig ist weiter, dass Lehramtsstudierende unter Kontrolle der Selbstkonzeptfacetten weniger demotiviert sind und ihre Studienwahl als stabiler einschätzen als Bachelorstudierende (vgl. Tab. 6 und 7 im Anhang).

Tab. 4 Standardisierte Regressionskoeffizienten zur Prädiktion der Studienzufriedenheit zu T2

\begin{tabular}{llllll}
\hline & Modell 1 & Modell 2 & Modell 3 & Modell 4 & Modell 5 \\
\hline Studiengang & $0,18^{*}$ & $0,17^{*}$ & $0,15^{*}$ & $0,12^{\mathrm{t}}$ & 0,01 \\
Allgemeines Selbstkonzept T1 & - & $0,13^{\mathrm{t}}$ & 0,15 & - & - \\
Schulbezogenes Selbstkonzept & - & - & $-0,13$ & - & - \\
T1 & & & & - \\
Hochschulbezogenes Selbstkon- & - & - & 0,07 & - & - \\
zept T1 & - & - & - & $0,47^{* * *}$ & $0,39^{* * *}$ \\
$\begin{array}{l}\text { Allgemeines Selbstkonzept T2 } \\
\text { Schulbezogenes Selbstkonzept }\end{array}$ & - & - & - & $-0,11^{\mathrm{t}}$ \\
$\begin{array}{l}\text { T2 } \\
\text { Hochschulbezogenes Selbstkon- } \\
\text { zept T2 }\end{array}$ & - & - & - & - & $0,30^{* * *}$ \\
$R^{2}$ & 0,033 & 0,049 & 0,067 & 0,250 & 0,334 \\
\hline
\end{tabular}

Studiengang: 1 - Bachelorstudium, 0 - Lehramtsstudium ${ }^{\mathrm{t}} p<0,10, * p<0,05, * * * p<0,001$ 


\section{Diskussion}

\subsection{Zusammenfassung}

Diverse Studien weisen darauf hin, dass sich die Domäne Mathematik beim Übergang in ein Mathematikstudium substanziell verändert (z. B. Gueudet 2008; Liebendörfer 2018). Diese Veränderung bewirkt potenziell eine Ausdifferenzierung des mathematischen Selbstkonzepts (Möller und Köller 2004). Um diese Ausdifferenzierung abzubilden, haben wir eine dreidimensionale Struktur des mathematischen Selbstkonzepts in eine allgemeine, eine universitäre und eine schulische Facette vorgeschlagen, operationalisiert und evaluiert.

Für diese vorgeschlagene Struktur des mathematischen Selbstkonzepts konnten wir Hinweise zu beiden Messzeitpunkten finden. Die Ausdifferenzierung des Selbstkonzepts zeigt sich, vermutlich basierend auf den Erwartungen der Studierenden (vgl. Rach et al. 2014), schon zu Beginn des Mathematikstudiums. Die Abnahme des allgemeinen mathematischen Selbstkonzepts im Laufe der ersten Studienwochen konnte repliziert werden (vgl. z. B. Geisler 2020; Rach und Heinze 2013). Die Trennung in drei Facetten ermöglicht darüber hinaus eine differenzierte Analyse: Während das schulische Selbstkonzept im Mittel stabil bleibt, sind das allgemeine und das universitäre mathematische Selbstkonzept rückläufig. Aufgrund der schon geringen Werte des universitären Selbstkonzepts zu Studienbeginn im Vergleich zum schulischen Selbstkonzept ist diese Entwicklung besonders zu beachten. Die Frage, die sich an die Entwicklungsverläufe der Facetten anschließt, ist, wodurch diese Entwicklungen bedingt sind. Dafür können wir an dieser Stelle Hypothesen aufstellen, die auf den Daten aufbauen und die dann in weiteren Studien überprüft werden müssen. Diese Hypothesen beziehen sich auf die verschiedenen Vergleichsmöglichkeiten der eigenen Leistung, die die Studierenden bei der Ausbildung und Anpassung ihres mathematischen Selbstkonzepts nutzen.

Der Rückgang des allgemeinen mathematischen Selbstkonzepts kann nicht allein auf sozialen Vergleichen im Sinne eines Big-Fish-Little-Pond-Effekts (Marsh 2005) basieren. Sonst wäre zu erwarten, dass sich der Rückgang auch auf das schulische Selbstkonzept auswirken müsste, welches sich jedoch in den ersten acht Wochen des Studiums nicht verändert. Weitere Vergleichsprozesse wirken entweder stabilisierend auf die schulische Facette oder stärker negativ auf die allgemeine Facette des mathematischen Selbstkonzepts. In jedem Fall scheint es zu einer Entkopplung der beiden Facetten zu kommen (vgl. Tab. 5 im Anhang). Obwohl ein direkter sozialer Vergleich zwischen Lehramts- und Bachelorstudierenden durch die unterschiedlichen Lehrveranstaltungen nur schwer möglich ist, nimmt das universitäre Selbstkonzept bei Lehramtsstudierenden stärker ab. Aufgrund unterschiedlicher mittlerer Vorleistungen beider Gruppen könnte dieses durch kriteriale Vergleiche anhand unterschiedlicher gezeigter Leistungen im Studium erklärt werden. Temporale Vergleichsmöglichkeiten würden vor allem die negative Entwicklung entweder allein des allgemeinen mathematischen Selbstkonzepts oder aller Selbstkonzeptfacetten erklären, denn die meisten Studierenden zeigen mehr Probleme beim Lösen mathematischer Aufgaben in der Studieneingangsphase als in der Schule (Liebendörfer und Göller 2016; Rach und Heinze 2013). Dimensionale Vergleiche zwischen 
der Schul- und Universitätsmathematik spielen möglicherweise eine geringere Rolle, denn der Vergleich der beiden Arten sollte zu einer negativen Kalibrierung des Selbstkonzepts in Bezug auf die universitäre Mathematik und zu einer positiven Kalibrierung des Selbstkonzepts in Bezug auf die Schulmathematik führen - letztere konnte aber nicht nachgewiesen werden. Bei Lehramtsstudierenden könnte der starke Rückgang des universitären Selbstkonzepts durch andere dimensionale Vergleiche erklärt werden: Bessere Leistungen im zweiten Fach, in der Fachdidaktik (vgl. Sorge et al. 2019) oder in den Bildungswissenschaften könnten im weiteren Studienverlauf zu einer negativen Kalibrierung des universitären mathematischen Selbstkonzepts beitragen. Insgesamt erklären verschiedene Vergleichsmöglichkeiten die Entwicklungen der Selbstkonzeptfacetten, wobei die Befundmuster weniger für eine überwiegende Rolle sozialer oder temporaler Vergleiche, sondern eher für kriteriale Vergleiche und möglicherweise dimensionale Vergleiche im Lehramtsstudium sprechen.

Hinweise auf die prädiktive Validität der Skalen wurden hinsichtlich des Studienerlebens berichtet. Durch die differenzierte Messung des mathematischen Selbstkonzepts konnten insgesamt zwischen $21 \%$ bis $33 \%$ der Varianz in den Maßen des Studienerlebens zur Semestermitte aufgeklärt werden. Das allgemeine und das universitäre mathematische Selbstkonzept sagen die Stabilität der Studienwahl und die Studienzufriedenheit positiv (vgl. Bernholt et al. 2018) und die Demotivation negativ vorher, wohingegen für das schulische mathematische Selbstkonzept keine signifikanten Effekte nachweisbar sind. Im Gegensatz zum Interesse (Kosiol et al. 2019) kann durch das Selbstkonzept zu Studienbeginn nur wenig auf das Erleben der Studieneingangsphase geschlossen werden; dagegen hängen die Selbstkonzeptfacetten zur Semestermitte mit dem Erleben eng zusammen. Somit scheinen die Entwicklung der Facetten in den ersten Semesterwochen und deren Ausprägung zur Semestermitte eine wichtige Rolle für das Erleben der Studieneingangsphase zu spielen. Auffällig ist, dass Lehramtsstudierende ihre Studienwahl unter Kontrolle der Selbstkonzeptfacetten als stabiler einschätzen als Fachstudierende. Lehramtsstudierende sind somit wohl in der Lage, eine geringere Studienzufriedenheit durch andere studienbezogene Überzeugungen zu kompensieren, um ihre Studienwahl zu stabilisieren.

\subsection{Limitationen}

Die Erhebung des universitären Selbstkonzepts zu Beginn eines Mathematikstudiums wirft die Frage auf, ob die Studierenden ihre eigenen Fähigkeiten zu einer Art von Mathematik einschätzen können, die sie gerade erst kennenlernen. Es ist bekannt, dass viele Studierende eine adäquate Vorstellung von universitärer Mathematik besitzen (Rach et al. 2014). Die Ergebnisse der Studie geben zudem keine Hinweise auf eine systematische, fehlerhafte Einschätzung dieser Selbstkonzeptfacette.

Weiterhin schränkt die Stichprobe selbst die Interpretationsreichweite der Ergebnisse ein. Für eine konfirmatorische Faktorenanalyse ist die Stichprobengröße recht klein. Zudem müssen wir von einer positiv selektierten Stichprobe ausgehen, denn die Stichprobenmortalität ist z. T. durch einen Modulabbruch bedingt. Für Personen, 
die das Mathematikmodul abbrechen, erwarten wir einen noch stärkeren Rückgang der Selbstkonzeptfacetten als hier berichtet.

Letztlich ist die Studie stark im Kontext des Mathematikstudiums verortet. Inwieweit die Struktur des Selbstkonzepts auf andere Studienfächer übertragbar ist, muss vor dem Hintergrund betrachtet werden, inwieweit sich andere Domänen beim Wechsel von der Schule in ein universitäres Studium in ähnlicher Weise verändern.

\subsection{Fazit}

Insgesamt zeigt sich, dass eine differenzierte Messung des mathematischen Selbstkonzepts möglich ist und interessante Einblicke in die Struktur, Entwicklung und Prädiktionskraft des mathematischen Selbstkonzepts ermöglicht. Die differenzierte Messung mit Hilfe von Facetten zeigt auf, dass sich wichtige Selbstkonzeptfacetten zu Studienbeginn rückläufig entwickeln. Solch einer negativen Entwicklung sollte im ersten Semester Rechnung getragen werden, indem beispielsweise stärker Aufgaben, die zum Kompetenzerleben beitragen, eingesetzt werden (vgl. z. B. Liebendörfer 2018). Denn aufgrund eines negativen universitären Selbstkonzepts, insbesondere beobachtet bei Lehramtsstudierenden, könnten Lerngelegenheiten zum zentralen Lerngegenstand der universitären Mathematik zu wenig wahrgenommen werden.

\section{Anhang}

Tab. 5 Korrelationen zwischen Selbstkonzeptfacetten zu Beginn und zur Mitte des ersten Semesters

\begin{tabular}{llll}
\hline & $\begin{array}{l}\text { Allgemeines Selbst- } \\
\text { konzept }\end{array}$ & $\begin{array}{l}\text { Schulisches Selbst- } \\
\text { konzept }\end{array}$ & $\begin{array}{l}\text { Universitäres Selbst- } \\
\text { konzept }\end{array}$ \\
\hline $\begin{array}{l}\text { Allgemeines Selbstkon- } \\
\text { zept }\end{array}$ & - & $0,47 * * *$ & $0,53 * * *$ \\
$\begin{array}{l}\text { Schulisches Selbstkon- } \\
\text { zept }\end{array}$ & $0,27 * * *$ & - & 0,10 \\
$\begin{array}{l}\text { Universitäres Selbst- } \\
\text { konzept }\end{array}$ & $0,44^{* * *}$ & $-0,07$ & - \\
\hline
\end{tabular}

Die Einträge über der Hauptdiagonalen sind die Korrelationen zum Beginn des ersten Semesters (T1), die Einträge unter der Diagonalen die Korrelationen zur Mitte des ersten Semesters (T2)

$* * * p<0,001$ 
Tab. 6 Standardisierte Regressionskoeffizienten zur Prädiktion der Demotivation zu T2

\begin{tabular}{|c|c|c|c|c|c|}
\hline & Modell 1 & Modell 2 & Modell 3 & Modell 4 & Modell 5 \\
\hline Studiengang & 0,08 & 0,10 & $0,13^{\mathrm{t}}$ & $0,15^{*}$ & $0,24 * * *$ \\
\hline Allgemeines Selbstkonzept T1 & - & $-0,21 * *$ & $-0,16^{\mathrm{t}}$ & - & - \\
\hline Schulisches Selbstkonzept T1 & - & - & 0,06 & - & - \\
\hline $\begin{array}{l}\text { Hochschulbezogenes Selbstkon- } \\
\text { zept T1 }\end{array}$ & - & - & $-0,15$ & - & - \\
\hline Allgemeines Selbstkonzept T2 & - & - & - & $-0,45 * * *$ & $-0,37 * * *$ \\
\hline Schulisches Selbstkonzept T2 & - & - & - & - & 0,08 \\
\hline $\begin{array}{l}\text { Hochschulbezogenes Selbstkon- } \\
\text { zept T2 }\end{array}$ & - & - & - & - & $-0,27 * * *$ \\
\hline$R^{2}$ & 0,007 & 0,050 & 0,071 & 0,207 & 0,271 \\
\hline
\end{tabular}

Studiengang: 1 - Bachelorstudium, 0 - Lehramtsstudium ${ }^{\mathrm{t}} p<0,10, * p<0,05, * * p<0,01, * * * p<0,001$

Tab. 7 Standardisierte Regressionskoeffizienten zur Prädiktion der Stabilität der Studienwahl zu T2

\begin{tabular}{llllll}
\hline & Modell 1 & Modell 2 & Modell 3 & Modell 4 & Modell 5 \\
\hline Studiengang & $-0,04$ & $-0,06$ & $-0,10$ & $-0,10$ & $-0,17^{*}$ \\
Allgemeines Selbstkonzept T1 & - & $0,23^{* *}$ & $0,18^{\mathrm{t}}$ & - & - \\
Schulisches Selbstkonzept T1 & - & - & $-0,12$ & - & - \\
Universitäres Selbstkonzept T1 & - & - & $0,20 *$ & - & - \\
Allgemeines Selbstkonzept T2 & - & - & - & $0,43^{* * *}$ & $0,37^{* * *}$ \\
Schulisches Selbstkonzept T2 & - & - & - & - & $-0,07$ \\
Universitäres Selbstkonzept T2 & - & - & - & - & $0,19^{*}$ \\
$R^{2}$ & 0,002 & 0,053 & 0,096 & 0,182 & 0,213 \\
\hline
\end{tabular}

Studiengang: 1 - Bachelorstudium, 0 - Lehramtsstudium ${ }^{\mathrm{t}} p<0,10, * p<0,05, * * p<0,01, * * * p<0,001$

Tab. 8 Übersicht über die verwendeten Skalen, die im Projekt SISMa neu entwickelt wurden

\section{Schulisches Selbstkonzept}

Was die Mathematik angeht, die ich in der Schule kennengelernt habe, bin ich ziemlich fit

Die Mathematik, die ich in der Schule kennengelernt habe, fällt mir leicht

Im Vergleich zu anderen Kommilitoninnen und Kommilitonen fällt mir die Mathematik, die ich an der Schule kennengelernt habe, leicht

\section{Universitäres Selbstkonzept}

Was die Mathematik angeht, die ich in der Hochschule kennengelernt habe, bin ich ziemlich fit

Die Mathematik, die an der Hochschule betrieben wird, fällt mir leicht

Im Vergleich zu anderen Kommilitoninnen und Kommilitonen fällt mir die Mathematik, die ich an der Hochschule kennengelernt habe, leicht

Funding Open Access funding enabled and organized by Projekt DEAL.

Open Access Dieser Artikel wird unter der Creative Commons Namensnennung 4.0 International Lizenz veröffentlicht, welche die Nutzung, Vervielfältigung, Bearbeitung, Verbreitung und Wiedergabe in jeglichem Medium und Format erlaubt, sofern Sie den/die ursprünglichen Autor(en) und die Quelle ordnungsgemäß nennen, einen Link zur Creative Commons Lizenz beifügen und angeben, ob Änderungen vorgenommen wurden. 
Die in diesem Artikel enthaltenen Bilder und sonstiges Drittmaterial unterliegen ebenfalls der genannten Creative Commons Lizenz, sofern sich aus der Abbildungslegende nichts anderes ergibt. Sofern das betreffende Material nicht unter der genannten Creative Commons Lizenz steht und die betreffende Handlung nicht nach gesetzlichen Vorschriften erlaubt ist, ist für die oben aufgeführten Weiterverwendungen des Materials die Einwilligung des jeweiligen Rechteinhabers einzuholen.

Weitere Details zur Lizenz entnehmen Sie bitte der Lizenzinformation auf http://creativecommons.org/ licenses/by/4.0/deed.de.

Interessenkonflikt S. Rach, S. Ufer und T. Kosiol geben an, dass kein Interessenkonflikt besteht.

\section{Literatur}

Bampili, A.-C., Zachariades, T., \& Sakonidis, C. (2017). The transition from high school to university mathematics: a multidimensional process. In T. Dooley \& G. Gueudet (Hrsg.), Proceedings of the Tenth Congress of the European Society for Research in Mathematics Education (S. 1985-1992). Dublin: DCU Institute of Education \& ERME.

Becker, M., \& Neumann, M. (2016). Context-related changes in academic self concept development: On the long-term persistence of big-fish-little-pond effects. Learning and Instruction, 45, 31-39.

Bernholt, A., Hagenauer, G., Lohbeck, A., Gläser-Zikuda, M., Wolf, N., Moschner, B., Lüschen, I., Klaß, S., \& Dunker, N. (2018). Bedingungsfaktoren der Studienzufriedenheit von Lehramtsstudierenden. Journal for Educational Research Online, 10(1), 24-51.

Blüthmann, I. (2012). Individuelle und studienbezogene Einflussfaktoren auf die Zufriedenheit von Bachelorstudierenden. Zeitschrift für Erziehungswissenschaft, 15(2), 273-303.

Bong, M., \& Skaalvik, E. M. (2003). Academic self-concept and self-efficacy: how different are they really? Educational Psychology Review, 15(1), 1-40.

Brandstätter, H., Grillich, L., \& Farthofer, A. (2002). Studienverlauf nach Studienberatung. Zeitschrift für Pädagogische Psychologie, 16(1), 15-28.

Cai, D., Viljaranta, J., \& Georgiou, G.K. (2018). Direct and indirect effects of self-concept of ability on math skills. Learning and Individual Differences, 61, 51-58.

Daskalogianni, K., \& Simpson, A. (2001). Beliefs overhang: the transition from school to university. Proceedings of the British Society for Research into Learning Mathematics, 21(2), 97-108.

Di Martino, P., \& Gregorio, F. (2019). The mathematical crisis in secondary-tertiary transition. International Journal of Science and Mathematics Education, 17(4), 825-843.

Dieter, M. (2012). Studienabbruch und Studienfachwechsel in der Mathematik. http://duepublico. uniduisburg-essen.de/servlets/DerivateServlet/Derivate-30759/Dieter_Miriam.pdf. Zugegriffen: 8. Dez. 2020.

Eccles, J.S., \& Wigfield, A. (2002). Motivational beliefs, values, and goals. Annual Review of Psychology, $53,109-132$.

Eccles, J.S., \& Wigfield, A. (2020). From expectancy-value theory to situated expectancy-value theory: A developmental, social cognitive, and sociocultural perspective on motivation. Contemporary Educational Psychology, 61, 101859.

Feng, X., Wang, J.-L., \& Rost, D.H. (2018). Akademische Selbstkonzepte und akademische Selbstwirksamkeiten: Interdependenzen und Beziehungen zu schulischen Leistungen. Zeitschrift für Pädagogische Psychologie, 32(1-2), 23-38.

Fleischer, J., Leutner, D., Brand, M., Fischer, H., Lang, M., Schmiemann, P., \& Sumfleth, E. (2019). Vorhersage des Studienabbruchs in naturwissenschaftlich-technischen Studiengängen. Zeitschrift für Erziehungswissenschaft, 22(5), 1077-1097.

Fredricks, J.A., \& Eccles, J.S. (2002). Children's competence and value beliefs from childhood through adolescence: Growth trajectories in two male-sex-typed domains. Developmental psychology, 38(4), $519-533$.

Geisler, S. (2020). Bleiben oder Gehen? Eine empirische Untersuchung von Bedingungsfaktoren und Motiven für frühen Studienabbruch und Fachwechsel in Mathematik (Dissertation). Universität Bochum.

Gueudet, G. (2008). Investigating the secondary-tertiary transition. Educational Studies in Mathematics, 67(3), 237-254.

Hefendehl-Hebeker, L. (2013). Doppelte Diskontinuität oder die Chance der Brückenschläge. In C. Ableitinger, J. Kramer \& S. Prediger (Hrsg.), Zur doppelten Diskontinuität in der Gymnasiallehrerbildung: Ansätze zu Verknüpfungen der fachinhaltlichen Ausbildung mit schulischen Vorerfahrungen und Erfordernissen (S. 1-15). Wiesbaden: Springer. 
Heublein, U., Hutzsch, C., Schreiber, J., Sommer, D., \& Besuch, G. (2009). Ursachen des Studienabbruchs in Bachelor- und in herkömmlichen Studiengängen: Ergebnisse einer bundesweiten Befragung von Exmatrikulierten des Studienjahres 2007/08. Hannover: HIS.

Jacobs, J.E., Lanza, S., Osgood, D. W., Eccles, J.S., \& Wigfield, A. (2002). Changes in children's selfcompetence and values: Gender and domain differences across grades one through twelve. Child development, 73(2), 509-527.

Jansen, M., Lüdtke, O., \& Robitzsch, A. (2020). Disentangling different sources of stability and change in students' academic self-concepts: An integrative data analysis using the STARTS model. Journal of Educational Psychology, 112(8), 1614-1631.

Kauper, T., Retelsdorf, J., Bauer, J., Rösler, L., Möller, J., \& Prenzel, M. (2012). PaLea - Panel zum Lehramtsstudium: Skalendokumentation und Häufigkeitsauszählungen des BMBF-Projektes. http://www. palea.uni-kiel.de/wp-content/uploads/2012/04/PaLea\%20Skalendokumentation\%204_\%20Welle. pdf. Zugegriffen: 5. März 2014.

Köller, O., Meyer, J., Saß, S., \& Baumert, J. (2019). New analyses of an old topic: Effects of intelligence and motivation on academic achievement. Journal für Bildungsforschung Online, 11(1), 166-189.

Köller, O., Trautwein, U., Lüdtke, O., \& Baumert, J. (2006). Zum Zusammenspiel von schulischer Leistung, Selbstkonzept und Interesse in der gymnasialen Oberstufe. Zeitschrift für Pädagogische Psychologie, 20(1/2), 27-39.

Kosiol, T., Rach, S., \& Ufer, S. (2019). (Which) mathematics interest is important for a successful transition to a university study program? International Journal of Science and Mathematics Education, 17(7), 1359-1380.

Liebendörfer, M. (2018). Motivationsentwicklung im Mathematikstudium. Wiesbaden: Springer Spektrum.

Liebendörfer, M., \& Göller, R. (2016). Abschreiben - Ein Problem in mathematischen Lehrveranstaltungen? In W. Paravicini \& J. Schnieder (Hrsg.), Hanse-Kolloquium zur Hochschuldidaktik der Mathematik 2014. Beiträge zum gleichnamigen Symposium am 7. \& 8. November 2014 an der Westfälischen Wilhelms-Universität Münster (S. 119-141). Münster: WTM-Verlag für wissenschaftliche Texte und Medien.

Lohbeck, A., Hagenauer, G., Mühlig, A., Moschner, B., \& Gläser-Zikuda, M. (2017). Prokrastination bei Studierenden des Lehramts und der Erziehungswissenschaften. Zeitschrift für Erziehungswissenschaft, 20, 521-536.

Marsh, H.W. (2005). Big-fish-little-pond effect in acedemic self-concept. Zeitschrift für Pädagogische Psychologie, 19(3), 119-127.

Marsh, H.W., Abduljabbar, A.S., Parker, P.D., Morin, A.J.S., Abdelfattah, F., Nagengast, B., Möller, J., \& Abu-Hilal, M.M. (2015). The internal/external frame of reference model of self-concept and achievement relations: Age-cohort and cross-cultural differences. American Educational Research Journal, 52(1), 168-202.

Marsh, H. W., Byrne, B. M., \& Shavelson, R. J. (1988). A multifaceted academic self-concept: Its hierarchical structure and its relation to academic achievement. Journal of Educational Psychology, 80, 366-380.

Marsh, H.W., Pekrun, R., Parker, P.D., Murayama, K., Guo, J., Dicke, T., \& Arens, A. K. (2019). The murky distinction between self-concept and self-efficacy-Beware of lurking jingle-jangle fallacies. Journal of Educational Psychology, 111(2), 331-353.

Möller, J., \& Köller, O. (2004). Die Genese akademischer Selbstkonzepte. Effekte dimensionaler und sozialer Vergleiche. Psychologische Rundschau, 55(1), 19-27.

Möller, J., \& Trautwein, U. (2015). Selbstkonzept. In E. Wild \& J. Möller (Hrsg.), Pädagogische Psychologie (S. 177-199). Berlin: Springer VS.

Möller, J., Pohlmann, B., Köller, O., \& Marsh, H. W. (2009). A meta-analytic path analysis of the internal/ external frame of reference model of academic achievement and academic self-concept. Review of Educational Research, 79(3), 1129-1167.

Nagel, K., \& Reiss, K. (2016). Zwischen Schule und Universität: Argumentation in der Mathematik. Zeitschrift für Erziehungswissenschaft, 19, 299-327.

Nagy, G., Watt, H. M. G., Eccles, J. S., Trautwein, U., Lüdtke, O., \& Baumert, J. (2010). The development of students' mathematics self-concept in relation to gender: Different countries, different trajectories? Journal of Research on Adolescence, 20(2), 482-506.

Neugebauer, M., Heublein, U., \& Daniel, A. (2019). Studienabbruch in Deutschland: Ausmaß, Ursachen, Folgen, Präventionsmöglichkeiten. Zeitschrift für Erziehungswissenschaft, 22(6), 1025-1046.

Parker, P. D., Schoon, I., Tsai, Y.-M., Nagy, G., Trautwein, U., \& Eccles, J. S. (2012). Achievement, agency, gender, and socioeconomic background as predictors of postschool choices: a multi-context study. Developmental Psychology, 48(6), 1629-1642. 
Rach, S. (2014). Charakteristika von Lehr-Lern-Prozessen im Mathematikstudium: Bedingungsfaktoren für den Studienerfolg im ersten Semester. Münster: Waxmann.

Rach, S., \& Heinze, A. (2013). Welche Studierenden sind im ersten Semester erfolgreich? Zur Rolle von Selbsterklärungen beim Mathematiklernen in der Studieneingangsphase. Journal für MathematikDidaktik, 34(1), 121-147.

Rach, S., \& Heinze, A. (2017). The transition from school to University in Mathematics: Which influence do school-related variables have? International Journal of Science and Mathematics Education, 15(7), 1343-1363.

Rach, S., Heinze, A., \& Ufer, S. (2014). Welche mathematischen Anforderungen erwarten Studierende im ersten Semester des Mathematikstudiums? Journal für Mathematik-Didaktik, 35(2), 205-228.

Schiefele, U., \& Jacob-Ebbinghaus, L. (2006). Lernermerkmale und Lehrqualität als Bedingungen der Studienzufriedenheit. Zeitschrift für Pädagogische Psychologie, 20(3), 199-212.

Schiefele, U., Moschner, B., \& Husstegge, R. (2002). Skalenhandbuch SMILE-Projekt. Bielefeld: Universität Bielefeld.

Schiefele, U., Streblow, L., \& Brinkmann, J. (2007). Aussteigen oder Durchhalten. Was unterscheidet Studienabbrecher von anderen Studierenden? Zeitschrift für Entwicklungspsychologie und Pädagogische Psychologie, 39(3), 127-140.

Shavelson, R. J., Hubner, J. J., \& Stanton, G. C. (1976). Self-concept: Validation of construct interpretations. Review of Educational Research, 46, 407-444.

Skaalvik, E. M., \& Skaalvik, S. (2002). Internal and external frames of reference for academic self-concept. Educational Psychologist, 37, 233-244.

Sorge, S., Keller, M., Neumann, K., \& Möller, J. (2019). Investigating the relationship between pre-service physics teachers' professional knowledge, self-concept, and interest. Journal of Research in Science Teaching, 56, 937-955.

Ufer, S., Rach, S., \& Kosiol, T. (2017). Interest in mathematics = interest in mathematics? What general measures of interest reflect when the object of interest changes. ZDM-Mathematics Education, 49(3), 397-409.

Wang, X. (2013). Why students choose STEM majors: Motivation, high school learning, and Postsecondary context of support. American Educational Research Journal, 50(5), 1081-1121.

Wigfield, A., Eccles, J. S., Mac Iver, D., Reuman, D. A., \& Midgley, C. (1991). Transitions during early adolescence: Changes in children's domain-specific self-perceptions and general self-esteem across the transition to junior high school. Developmental psychology, 27(4), 552-565.

Wild, K.-P. (2005). Individuelle Lernstrategien von Studierenden. Konsequenzen für die Hochschuldidaktik und die Hochschullehre. Beiträge zur Lehrerbildung, 23(2), 191-206.

Winter, H. (1996). Mathematikunterricht und Allgemeinbildung. Mitteilungen der Gesellschaft für Didaktik der Mathematik, 61, 37-46. 